diese Näherung sicher nur dann, wenn der Energieverlust durch Strahlung hinreichend klein ist. Die Dämpfung durch Stöße steigt mit der Ordnungszahl des Schwingungstyps an und nimmt ab mit dem statischen Magnetfeld. Die Dichte $\sigma$ der Eigenwerte (Zahl pro Frequenzbereich) steigt wegen der $n_{\mathrm{r}}{ }^{-1}$. Abhängigkeit (50) mit der Teilchendichte und sinkt mit dem Magnetfeld.

$$
\sigma=\text { const } \cdot N^{1 / 2} H_{0}^{-1} .
$$

Die Gesamtzahl der Eigenwerte unterhalb der Ionenresonanz ist aber unabhängig vom Magnetfeld, da der gesamte zur Verfügung stehende Frequenzbereich mit dem Magnetfeld zunimmt, im selben Maße wie die Eigenwertdichte abnimmt.

\section{Freie Schwingungen einer Plasmaplatte in einem Magnetfeld}

Das dem hier vorliegenden zylindrischen Problem mit azimutalen Strömen und axialem Magnetfeld entsprechende ebene Gebilde ist ein in $y$ - und $z$-Richtung unbegrenzter plattenförmiger Plasmabereich,

$$
-x_{0} \leqq x \leqq x_{0},
$$

welcher einem homogenen statischen Magnetfeld in $z$-Richtung ausgesetzt ist. In den beiden Begrenzungsebenen $x=x_{0}$ und $x=-x_{0}$ fließen Wechselströme in $y$-Richtung im Gegentakt, welche ebene elektromagnetische Wellen anregen. Der Brechungsindex des Mediums wird für diese Polarisation $\left(E_{y}, H_{z}\right)$ wieder durch (16) gegeben. (Vgl. I.)

Bei verschwindenden Strömen erhält man als Gleichung für die Eigenfrequenzen $\omega$ des Systems

$$
\omega=\frac{c}{2 i n(\omega) x_{0}} \cdot \ln \frac{n(\omega)-1}{n(\omega)+1} .
$$

Wegen der Vieldeutigkeit des Logarithmus treten wieder Oberschwingungen auf, welche aber nicht streng äquidistant liegen, da sich $n$ mit $\omega$ ändert.

\title{
Die Impedanz einer Spule mit einem Plasma als Dielektrikum
}

\author{
Von Klaus Körper \\ Aus dem Max-Planck-Institut für Physik, Göttingen * \\ (Z. Naturforschg. 15 a, 226-235 [1960]; eingegangen am 17. Januar 1960)
}

\begin{abstract}
From the basic equations of magneto-hydrodynamics the energy conservation theorem for plasmas is derived for events periodic in time. In addition to the terms known from vacuum electrodynamics, there are the kinetic energies of the electrons and ions, and a term due to the oscillations of the electrons and ions in the magnetic field. - With the help of the solution for the radially oscillating cylinder, the impedance of a coil containing the plasma is derived from general energy considerations. The impedance [eq. (31)] is discussed and its mean value in the range between two eigenfrequencies of the plasma cylinder is given.
\end{abstract}

In einer früheren Arbeit ${ }^{1,2}$ wurden die Lösungen der auf einer Zwei-Flüssigkeits-Theorie beruhenden Grundgleichungen des Plasmas ${ }^{3}$ für einen in einem axialen Magnetfeld radial schwingenden homogenen unendlich langen Plasmazylinder hergeleitet. Hiermit läßt sich auch die durch die kollektive Schwingung der geladenen Plasmamaterie bedingte makroskopische Ausstrahlung ins äußere umgebende Vakuum beschreiben.

Die von strahlenoptischen Modellvorstellungen der Wellenausbreitung herrührende begriffliche Schwierigkeit der Unterscheidung: absorbierte und das endliche Plasmavolumen durchdringende Energie oder

* Seit Herbst 1958 in München.

1 K. Körper, Z. Naturforschg. 12 a, 815 [1957]. Im folgenden als I bezeichnet. aufgenommene und reflektierte Energie entfällt im Rahmen einer feldmäßigen Behandlung. Hier lassen sich die betreffenden Leistungen aus dem resultierenden Wellenfeld durch Oberflächenintegrale des Poynting-Vektors über geeignet gelegte Hüllflächen gewinnen.

\section{Energetische Betrachtungen}

Bei der üblichen Art der Interpretation komplexer Größen hat nur ihr Realteil unmittelbare physikalische Bedeutung. Komplexe Größen werden nur der rechnerischen Einfachheit halber bevorzugt.

\footnotetext{
2 K. KöRPER, Z. Naturforschg. 15 a, 220 [1960]. Im folgenden mit II bezeichnet.

3 A. Schlüter, Z. Naturforschg. 5 a, 721 [1950].
} 
Wenn zwei reelle sinusförmig periodische Größen

$$
a=A \cdot \cos \omega t, \quad b=B \cdot \cos (\omega t-\varphi)
$$

mit der gegenseitigen Phasenverschiebung $\varphi$ vorliegen, dann zerfällt das Produkt

$$
a b=A B\left[\cos \varphi \cos ^{2} \omega t+\sin \varphi \cdot \frac{1}{2} \cdot \sin (2 \omega t)\right]
$$

in einen pulsierenden positiv definiten Anteil und einen um den Nullpunkt oszillierenden Anteil. Im Zeitmittel über eine Periode liefert nur der erste Term einen Beitrag. Interpretiert man das Produkt $a b$ als die „Leistung“ der Größen $a$ und $b$, dann folgt für den allgemein als Wirkleistung bezeichneten ersten Term im Zeitmittel

$$
\overline{a b}^{t}=\frac{1}{2} \cdot A B \cdot \cos \varphi .
$$

$\cos \varphi$ ist in der Schwingungsphysik als „Leistungsfaktor" eingeführt. Bei komplexer Schreibweise stellen sich die Größen $a$ und $b$ dar als

$$
a=A \cdot e^{i \omega t}, \quad b=B \cdot e^{i(\omega t-\varphi),}
$$

wobei $A$ und $B$ reell sind. Für den Ausdruck

$$
P=\frac{1}{2} a b^{*}
$$

ergibt sich dann $\quad P=\frac{1}{2} A B \cdot e^{i \varphi}$.

Ein Vergleich mit (2) zeigt, daß der Realteil von $P$ gerade den Zeitmittelwert der Wirkleistung darstellt nud der Imaginärteil die Amplitude der mit doppelter Frequenz oszillierenden, im Zeitmittel verschwindenden Blindleistung.

Daher wird im folgenden die Bildung (5) immer dann von Bedeutung sein, wenn Ausdrücke auftreten, die in den zeitlich periodischen komplexen Feldgrößen quadratisch sind.

Zur Herleitung des Energiesatzes des Plasmas bei komplexen Feldgrößen multipliziert man von den Grundgleichungen

$$
\begin{aligned}
\operatorname{rot} \mathfrak{S} & =\frac{4 \pi}{c} \cdot \dot{\mathrm{j}}+i \frac{\omega}{c} \cdot \mathfrak{F} \\
\operatorname{rot} \mathfrak{F} & =\quad-i \frac{\omega}{c} \mathfrak{S} \\
\varrho i \omega \mathfrak{b} & =\frac{1}{c} \cdot\left[\mathrm{j} \times \mathfrak{S}_{0}\right] \\
\frac{4 \pi}{\omega_{\mathrm{p}}{ }^{2}}(i \omega+\gamma) \cdot \dot{\mathrm{j}} & =\mathfrak{E}+\frac{1}{c} \cdot\left[\mathfrak{b} \times \mathfrak{S}_{0}\right]-\frac{m_{\mathrm{i}}-m_{\mathrm{e}}}{e} i \omega \mathfrak{v},
\end{aligned}
$$

die zweite mit $-\mathfrak{S}^{*}$, die erste, konjugiert genommen, mit $\&$ und addiert beide. Multiplikation der vierten mit $j^{*}$ und Eliminierungen liefern unter Berücksichtigung bekannter Vektorumformungen

$$
\begin{aligned}
& -\operatorname{div} \cong=\frac{4 \pi}{\omega_{\mathrm{p}}^{2}} \cdot \gamma \cdot\left|\frac{\mathrm{j}^{2}}{2}\right| \\
& +2 i \omega\left\{\frac{1}{8 \pi}\left(\left|\frac{\mathfrak{H}^{2}}{2}\right|-\left|\frac{\mathfrak{F}^{2}}{2}\right|\right)+\frac{2 \pi}{\omega_{\mathrm{p}}^{2}} \cdot\left|\frac{\mathrm{j}^{2}}{2}\right|-\frac{\varrho}{2} \cdot\left|\frac{\mathfrak{v}^{2}}{2}\right|\right. \\
& \left.-\frac{m_{\mathrm{i}}-m_{\mathrm{e}}}{e c \cdot \varrho}\left(\mathfrak{S}_{0} \cdot\left[\dot{\mathrm{j}} \times \mathrm{i}^{*}\right]\right)\right\}
\end{aligned}
$$

mit dem Poynting-Vektor

$$
\widetilde{S}=\frac{c}{4 \pi} \cdot \frac{1}{2}\left[\left(\tilde{E} \times \mathfrak{S}^{*}\right] .\right.
$$

Die Größen $\mathfrak{F}, \mathfrak{H}, \mathfrak{v}$, I stellen Amplituden der Schwingung dar, $\mathfrak{E} / \sqrt{2}, \mathfrak{S} / \sqrt{2}, \mathfrak{v} / \sqrt{2}, \mathrm{i} / \sqrt{2}$ bekanntlich die Effektivwerte.

Der komplexe Energiesatz des Plasmas besagt: der lokale Schwund der Strahlungsdichte enthält im Zeitmittel als Wirkanteil die der Stoßfrequenz $\gamma$ proportionale Joulesche Erwärmung des Plasmas und als Blindleistung neben der Differenz zwischen mittlerer magnetischer und elektrischer Feldenergiedichte wie im Vakuum auch noch die Differenz zwischen den kinetischen Energiedichten zwischen Elektronen und Ionen. Außerdem tritt noch ein rein imaginärer Term auf, der auf der Wechselwirkung der bewegten Ladungsträger mit dem statischen Magnetfeld beruht.

Zur Anwendung des Energiesatzes (11) auf das vorliegende Problem hat man die stromführende Schicht bei $r=R$ durch zwei eng anliegende Hüllflächen I und II nach außen $(r>R)$ und nach innen $(r<R)$ hin abzuschließen. Auf diese Weise entstehen zwei voneinander getrennte Bereiche, in welche von der als Antenne wirkenden Stromschicht auf dem Zylindermantel elektromagnetische Wellen gesandt werden. Im äußeren Bereich gilt dann die übliche Vakuumelektrodynamik, im inneren die Plasmagrundgleichungen (7) bis (10). Die Leistungen in den beiden Bereichen erhält man dann durch Ober-

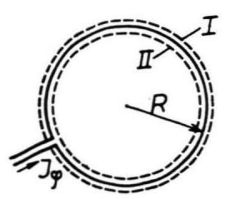

Abb. 1. Lage der Hüllflächen.

flächenintegrale über die Radialkomponente des Poynting-Vektors. Wegen der Unabhängigkeit aller Feldgrößen vom Azimutwinkel $\varphi$ läßt sich die Integration leicht durchführen. Die Bildung (5) liefert dann den Zeitmittelwert der komplexen Scheinleistung pro Längeneinheit des Plasmazylinders. 
Aus der Lösung der Maxwellschen Gleichungen im Vakuum [vgl. I]

$$
\begin{array}{r}
E_{\varphi}(\mathrm{r}, t)=\alpha_{2} \cdot H_{1}{ }^{(2)}(k r) e^{i \omega t} \\
H_{z}(\mathrm{r}, t)=i \alpha_{2} \cdot H_{0}^{(2)}(k r) e^{i \omega t} \\
\alpha_{2}=\frac{4 \pi}{c} \hat{I}_{\varphi} \cdot \frac{J_{1}(n k R)}{J_{0}(n k R) \cdot H_{1}(2)(k R)-n \cdot J_{1}(n k R) \cdot H_{0}(2)(k R)}
\end{array}
$$

gewinnt man die Verluststrahlung ins Unendliche

$$
P_{1}=\int_{\mathrm{I}} \widetilde{\Xi}_{\mathrm{r}} \mathrm{d} f=\frac{c R}{4} E_{\varphi} \cdot H_{z}{ }^{*}=\frac{c R}{4} \cdot\left|\alpha_{2}\right|^{2} \cdot(W-i V) \text {. }
$$

Hierbei bedeuten $W$ die $W_{\text {ronskische Determinante }}$

$$
W=J_{1}(k R) \cdot N_{0}(k R)-J_{0}(k R) \cdot N_{1}(k R) \equiv \frac{2}{\pi} \cdot \frac{1}{k R}
$$

und $V$ den Ausdruck

$$
V=J_{0}(k R) \cdot J_{1}(k R)+N_{0}(k R) \cdot N_{1}(k R) .
$$

Mit den Lösungen im Plasma

$$
\begin{gathered}
E_{\varphi}(\mathrm{r}, t)=a \cdot J_{1}(n k r) \cdot e^{i \omega t}, \\
H_{z}(\mathrm{r}, t)=a i n \cdot J_{0}(n k r) \cdot e^{i \omega t}, \\
a=\frac{4 \pi}{c} \hat{I}_{\varphi} \cdot \frac{H_{1}(2)(k R)}{J_{0}(n k R) \cdot H_{1}(2)(k R)-n \cdot J_{1}(n k R) \cdot H_{0}{ }^{(2)}(k R)}
\end{gathered}
$$

ergibt sich die Scheinleistung im Plasma

$P_{2}=-\int_{\text {II }} \widetilde{S}_{\mathrm{r}} \mathrm{d} f=\frac{c R}{4}|a|^{2} i n^{*} J_{1}(n k R) J_{0}{ }^{*}(n k R)$.

Der in den Wellenamplituden $a(21)$ und $\alpha_{2}$ (15) und damit in den Leistungen $P_{1}$ und $P_{2}$ noch als frei vorgebbar betrachtete Spulenstrom $\hat{I}_{\varphi}$ wird durch die äußere Schaltung festgelegt. Der Faktor $n^{*}$ in $P_{2}$ deutet darauf hin, daß für die Absorption der elektromagnetischen Strahlung im Plasma die Extrema von $n_{\mathrm{i}}$ maßgebend sind.

Wegen $V>0$ ist die Blindleistung der Strahlung ins Vakuum negativ.

Da in ebenen Wellen im Vakuum die elektrische Feldstärke gleich der magnetischen ist, hat dort das anregende ebene Stromsystem keine Blindleistung aufzubringen. Das folgt aus dem Energiesatz (11) mit $\mathfrak{v}=\mathrm{i}=0$.

Durch die zylindrische Krümmung aber wird die Symmetrie zwischen elektrischem und magnetischem Feld gestört. Die Biegung der elektrischen Feldlinien in der Zylinderwelle führt zu einer kapazitiven Belastung der Antenne. - Axiale Ströme, welche eine Welle mit der um $\pi / 2$ gedrehten $E_{z}-H_{\varphi}$-Polarisation anregen, werden induktiv belastet. Man erhält nämlich dann aus dem
Wellenfeld im Vakuum

$$
\begin{gathered}
E_{z}(\mathrm{r}, t)=-i \beta \cdot H_{0}{ }^{(2)}(k r) \cdot e^{i \omega t}, \\
H_{\varphi}(\mathrm{r}, t)=\beta \cdot H_{1}{ }^{(2)}(k r) \cdot e^{i \omega t}, \\
\beta=\frac{4 \pi}{c} \hat{I}_{z} \cdot \frac{J_{0}(m k R)}{J_{0}(m k R) \cdot H_{1}{ }^{(2)}(k R)-m \cdot J_{1}(m k R) \cdot H_{0}{ }^{(2)}(k R)}
\end{gathered}
$$

für die mittlere Strahlung [vgl. (16)]:

$$
P_{1}^{\prime}=\frac{c R}{4} \cdot|\beta|^{2} \cdot(W+i V) .
$$

Aus dem Energiesatz folgt auch, daß man die räumliche Verteilung der Dichte der dissipierten Energie (Joulesche Wärme) unmittelbar aus den Feldvektoren herleiten kann [(11), (12), (19), $(20)]$ :

$u(r)=\Re e\{-\operatorname{div} \Im\}$

$$
=\frac{c}{8 \pi}|a|^{2} k\left|J_{1}(n k r)\right|^{2} \mathfrak{J m}\left\{-n^{2}\right\} .
$$

Diese Verteilung entspricht der Verteilung der elektrischen Feldenergiedichte.

Da der Faktor $\mathfrak{J}\left(n^{2}\right)=\left(n^{2}\right)_{\mathrm{i}}$ der Stoßfrequenz $\gamma$ proportional ist, kann man im Rahmen einer Linearisierung alle anderen Größen in (27) von $\gamma$ unabhängig annehmen. Diese Näherung ist dann zulässig, wenn die Dämpfung der Welle durch Thermalisierung auf ihrem Wege durch das Plasma so gering ist, daß praktisch eine homogene Aufheizung stattfindet. - Im ebenen Falle einer exponentiell abklingenden Welle in einem absorbierenden Medium würde diese Näherung bedeuten, daß die Entwicklung

$$
\frac{\mathrm{d}}{\mathrm{d} x}\left(e^{-\varkappa x}\right)=-\varkappa \cdot(1-\varkappa \cdot x \pm \ldots)
$$

nach dem ersten Glied abgebrochen werden kann. Die Energieabnahme der Welle durch die Absorption wird in dieser Näherung vernachlässigt.

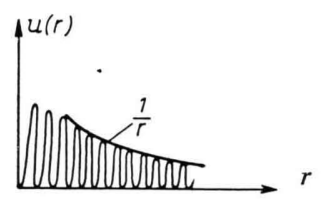

Abb. 2. Räumliche Verteilung der Energiedichte.

Da sich stehende Wellen im Plasmazylinder ausbilden, findet eine Aufheizung nur in einzelnen, sehr dicht liegenden Ringzonen der Breite $\Delta r=\frac{\pi}{2} \cdot \frac{1}{n k}$ statt. Wegen des $r^{-1}$-Abfalls der BesseL-Funktionen und damit der Energiedichte $u(r)$ wird in jeder Zone dieselbe Energie aufgenommen. 
Der Anstieg der Energiedichte in den inneren Ringzonen wird bedingt durch die Überlagerung der ebenen Elementarwellen, aus denen man sich Zylinderwellen zusammengesetzt denken kann ${ }^{4}$.

\section{Die Eingangsimpedanz des äußeren Strom- systems}

\section{a) Plasma grenzt unmittelbar an die Strombahn}

Den Spulenwiderstand, der Strahlung und Absorption berücksichtigt, gewinnt man am einfachsten aus einer energetischen Betrachtung.

Die gesamte (komplexe) Scheinleistung der Hochfrequenzspule erhält man durch Integration der Radialkomponente des Poynting-Vektors über beide die stromführende Schicht innen und außen umschließende Hüllflächen. Man erhält pro Längeneinheit des Plasmazylinders

$$
P=\int_{\mathrm{I}+\mathrm{II}} \Im_{\mathrm{I}} \mathrm{d} f=\frac{c}{8 \pi} \cdot \int_{\mathrm{I}+\mathrm{II}} E_{\varphi} \cdot H_{z}^{*} \mathrm{~d} f=P_{1}+P_{2} .
$$

Berücksichtigung des stetigen Überganges des elektrischen Feldes durch die Stromschicht und der Normalenrichtung der zweiblättrigen Hüllfläche liefert dann

$$
P=\frac{c}{8 \pi} \cdot 2 \pi R \cdot E_{\varphi}(R)\left[H_{z}{ }^{*}(R+0)-H_{z}{ }^{*}(R-0)\right] .
$$

Über die Sprungbedingung des magnetischen Feldes läßt sich der Strom $\hat{I}_{\varphi}$ pro Längeneinheit einführen. Wenn man den Wert (19) und (21) für das elektrische Feld einsetzt, dann folgt für die gesamte Leistung der HF-Spule pro Längeneinheit

$$
\begin{aligned}
P=( & -i) \cdot \frac{4 \pi}{c} \cdot 2 \pi R \cdot \frac{\left|\hat{I}_{\varphi}\right|^{2}}{2} \\
& \cdot \frac{H_{1}(2)(k R) \cdot J_{1}(n k R)}{J_{1}(n k R) \cdot H_{0}(2)(k R)-n J_{0}(n k R) \cdot H_{1}(2)(k R)} .
\end{aligned}
$$

Besitzt die Spule die Windungsdichte $v$ (Zahl der Windungen pro Längeneinheit), dann ist der Flächenstrom $\hat{I}_{\varphi}$ in der elektrodynamischen Wirkung einer $v$-fachen Windung des linearen Stromes $\mathfrak{J}=\hat{I}_{\varphi} / v$ äquivalent. Nach Abspalten des Faktors $\frac{1}{2}|\mathfrak{J}|^{2}$ erhält man für die Eingangsimpedanz einer mit einem Plasma im Magnetfeld belasteten Spule der Länge $l$ :

$\Re=(-i) M_{I_{1}(n k R) \cdot H_{0}(2)(k R)-n J_{0}(n k R) \cdot H_{1}(2)(k R)}$
Hierin bedeutet

$$
M=\frac{4 \pi}{c} \cdot 2 \pi R \cdot l \nu^{2} .
$$

Den Sonderfall der Vakuumspule $(n=1)$ kann man hieraus leicht herleiten: Wenn die Vakuumwellenlänge $\lambda$ groß ist gegen den Spulenumfang $(2 \pi R \ll \lambda)$ bzw. $(k R \ll 1)$, lassen sich in (31) die Zylinderfunktionen für kleine Argumente entwickeln. Man erhält in diesem quasistationären Grenzfall den rein induktiven Widerstand

$$
\mathfrak{R}=i \omega L \quad \text { mit } \quad L=\frac{4 \pi}{c^{2}} \cdot \pi R^{2} \cdot l \cdot v^{2} .
$$

$L$ ist bekanntlich die Induktivität einer Spule der Länge $l$ und der Windungszahl $l v$ im Gauss schen Maßsystem. Für das elektromagnetische Feld folgt aus (19), (20) und (13), (14) im Grenzfall kleiner Frequenzen $(\omega \rightarrow 0)$ im Innenraum der Spule $(r \leqq R)$

$$
\begin{aligned}
E_{\varphi}(\mathrm{r}, t) & \rightarrow-i \frac{4 \pi}{c} \hat{I}_{\varphi} \frac{k r}{2} e^{i \omega t}, \\
H_{z}(\mathrm{r}, t) & \rightarrow \frac{4 \pi}{c} \hat{I}_{\varphi} e^{i \omega t}
\end{aligned}
$$

und im Außenraum $(r \geq R)$ in derselben Näherung

$$
\begin{aligned}
& E_{\varphi}(\mathrm{r}, t) \rightarrow-i \frac{4 \pi}{c} \hat{I}_{r} \frac{k R}{2} \frac{R}{r} e^{i \omega t}, \\
& H_{z}(\mathrm{r}, t) \rightarrow 0 .
\end{aligned}
$$

Die Bauart der Gl. (31) legt es nahe, den gesamten Plasmawiderstand $\Re \mathrm{zu}$ interpretieren als den

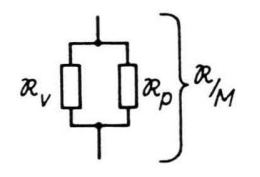

Abb. 3. Zusammensetzung der Feldwellenwiderstände zur Spulenimpedanz.

Widerstand einer Parallelschaltung (Abb. 3) aus dem Feldwellenwiderstand

$$
\Re_{\mathrm{V}}(R)=\left(\frac{E_{\varphi}(R)}{H_{z}(R)}\right)_{\mathrm{V}}=\frac{H_{1}(2)(k R)}{i H_{0}(2)(k R)}
$$

für nach außen im Vakuum ins Unendliche laufende Zylinderwellen und dem Feldwellenwiderstand

$$
\Re_{\mathrm{P}}(R)=-\left(\frac{E_{\varphi}(R)}{H_{z}(R)}\right)_{\mathrm{P}}=\frac{i J_{1}(n k R)}{n J_{0}(n k R)}
$$

für stehende Wellen im Plasma. - Feldwellenwiderstände hängen von dem Medium und von der Geometrie der Welle ab; außerdem hat man Vorzeichen einzuführen. - In dieser Darstellung ist $M$ ein Faktor, der die Kopplung des elektromagnetischen Feldes an das Stromsystem beschreibt.

4 A. Sommerfeld, Vorl. über Theoret. Physik, Bd. VI, Leipzig 1947. 
Bei den praktisch vorkommenden Plasmen hängt das Verhalten von $\Re$ entscheidend von der Betriebsfrequenz $\omega$ und den Plasmadaten ab. Oberhalb der Ionenresonanz ist der Brechungsindex im wesentlichen imaginär (vgl. II, Abb. 4 und 5, und I, Abb. 3). Für $\left|n_{\mathrm{i}} k R\right| \gg 1$ steigen in (31) die Bessel-Funktionen exponentiell an ${ }^{5}$ :

$$
\begin{aligned}
& J_{1}(i x) \rightarrow-i e^{-x} / \sqrt{2 \pi i x} \\
& J_{0}(i x) \rightarrow e^{-x} / \sqrt{2 \pi i x}
\end{aligned}
$$

für

$$
|x| \gg 1, \quad x<0 \text {. }
$$

Daher bleibt

$$
\Re=(-i) M \frac{H_{1}^{(2)}(k R)}{H_{0}(2)(k R)-i n \cdot H_{1}^{(2)}(k R)} .
$$

Diese Beziehung gilt auch noch in und dicht unterhalb der Ionenresonanz.

Weiter unterhalb der Ionenresonanz ist $n_{\mathrm{r}} \gg\left|n_{\mathrm{i}}\right|$. Hier wird der Widerstand $\Re$ und die Amplituden $a$ und $\alpha_{2}$ (21), (15) fastperiodische Funktionen mit der Periode $\Delta\left(n_{\mathrm{r}} k R\right)=\pi$. Ausgeprägte Maxima von $\Re$ treten bei Werten $n(\omega) \omega(R / c)$ auf, $\omega$ reell, die in der Nähe einer der komplexen Lösungen der Eigenwertgleichung

$$
\begin{aligned}
& \mathfrak{N}^{(2)}(\omega) \equiv J_{1}\left(n(\omega) \omega \frac{R}{c}\right) H_{0}{ }^{(2)}\left(\omega \frac{R}{c}\right) \\
& -n(\omega) J_{0}\left(n(\omega) \omega \frac{R}{c}\right) H_{1}{ }^{(2)}\left(\omega \frac{R}{c}\right)=0
\end{aligned}
$$

für einen im Vakuum frei schwingenden Plasmazylinder liegen [vgl. II, Gl. (41)]. Diese Maxima treten auf, wenn das Magnetfeld der stehenden Welle (20) in der Nähe des Plasmarandes klein wird; Minima dagegen, wenn das elektrische Feld (19) am Plasmarand ein Minimum hat.

Im Rahmen des Wellenwiderstandkonzeptes kann man den Grund dieser Welligkeit in einer mehr oder minder guten Anpassung des Wellenwiderstandes $\Re_{P}$ des Plasmas (37) an den des umgebenden Vakuums $\Re_{\mathrm{V}}$ (36) sehen, denn die linke Seite der Gl. (41) ist im wesentlichen die Summe der Wellenwiderstände. (Anpassung liegt vor, wenn die Summe der Widerstände verschwindet.)

Entscheidend ist, daß die starke Welligkeit nicht von der etwas künstlichen Annahme eines scharfen

5 Magnus-Oberhettinger, Formeln u. Sätze für die speziellen Funktionen der Mathematischen Physik. Springer-Verlag, Berlin 1943.
Plasmarandes herrührt, sondern von der Größe des Brechungsindexes. Eine nähere Untersuchung zeigt, $\mathrm{da} ß$ auch bei einem inhomogenen Plasma mit einem glockenförmigen Verlauf (Abb. 4) des Brechungs. indexes

$$
n^{2}(r)=\frac{n^{2}+\left(r / r_{0}\right)^{2}}{1+\left(r / r_{0}\right)^{2}}
$$

dieselben Schwierigkeiten auftreten. Falls $n_{\mathrm{r}} \gg\left|n_{\mathrm{i}}\right|$, d. h. unterhalb der Ionenresonanz, entspricht diesem Verlauf eine Verteilung der Teilchendichte von

$$
N(r)=\operatorname{const}\left[n^{2}(r)-1\right] .
$$

Für $r / r_{0} \gg 1$ wird die Wellengleichung mit dem Brechungsindex (42) durch Zylinderfunktionen der Ordnung $v=i \sqrt{\left(n k r_{0}\right)^{2}-1}$ und dem Argument $k r$ gelöst. Die asymptotische Darstellung der Bessel-Funktion mit kleinem Argument und hoher komplexer

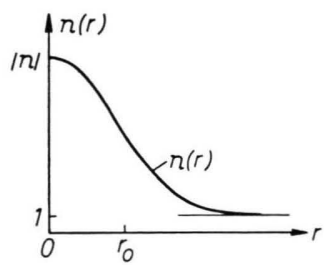

Abb. 4. Brechungsindex eines inhomogenen Plasmas.

Ordnung ${ }^{6}$ zeigt, daß wieder schnelle Oszillationen bei kleiner Variation der Frequenz, der Dichte oder des Radius auftreten, wenn $\left|n k r_{0}\right| \gg 1$ ist. Hier spielt jetzt die Halbwertsbreite $r_{0}$ des Abfalls vom Maximalwert $n$ auf den Vakuumwert 1 für $r \rightarrow \infty$ dieselbe Rolle wie der Radius $R$ des Plasmazylinders mit scharfem Rand.

Wegen $|n k R| \gg 1$ kann man in Gl. (31) die BESSEL-Funktionen asymptotisch entwickeln ${ }^{5}$.

$$
\begin{aligned}
& J_{0}(z) \approx \sqrt{\frac{2}{\pi z}} \cdot \cos \left(z-\frac{\pi}{4}\right), \\
& J_{1}(z) \approx \sqrt{\frac{2}{\pi z}} \cdot \sin \left(z-\frac{\pi}{4}\right) .
\end{aligned}
$$

Nach Aufspalten in Real- und Imaginärteil treten dann $x_{\mathrm{r}}=n_{\mathrm{r}} k R-\frac{\pi}{4}$ als Argument der trigonometrischen Funktionen, $x_{\mathrm{i}}=n_{\mathrm{i}} k R$ als Argument der Hyperbelfunktionen auf. Man findet

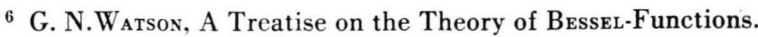
Cambridge, At the University Press 1952. 


$$
\begin{aligned}
& \Re=\frac{M}{N}\left\{\left[W \cdot\left(\sin ^{2} x_{\mathrm{r}} \cdot \operatorname{cof}^{2} x_{\mathrm{i}}+\cos ^{2} x_{\mathrm{r}} \cdot \sin ^{2} x_{\mathrm{i}}\right)-\alpha \cdot\left(-n_{\mathrm{i}} \cos x_{\mathrm{r}} \cdot \sin x_{\mathrm{r}}+n_{\mathrm{r}} \cdot \operatorname{Co}\left\lceil x_{\mathrm{i}} \cdot \operatorname{Sin} x_{\mathrm{i}}\right)\right]\right.\right. \\
& -i \cdot\left[V \cdot\left(\sin ^{2} x_{\mathrm{r}} \cdot \operatorname{Sof}^{2} x_{\mathrm{i}}+\cos ^{2} x_{\mathrm{r}} \cdot \operatorname{Sin}^{2} x_{\mathrm{i}}\right)-\alpha \cdot\left(n_{\mathrm{i}} \operatorname{So}\left\{x_{\mathrm{i}} \operatorname{Sin} x_{\mathrm{i}}+n_{\mathrm{r}} \cdot \cos x_{\mathrm{r}} \cdot \sin x_{\mathrm{r}}\right)\right]\right\}
\end{aligned}
$$

mit den Abkürzungen

$$
\begin{aligned}
& \alpha=J_{1}{ }^{2}(k R)+N_{1}{ }^{2}(k R)=\left|H_{1}{ }^{(2)}(k R)\right|^{2}, \\
& \beta=J_{0}{ }^{2}(k R)+N_{0}{ }^{2}(k R)=\left|H_{0}{ }^{(2)}(k R)\right|^{2} .
\end{aligned}
$$

\section{Der Nenner}

$$
N=\boldsymbol{a} \cdot \cos ^{2} x_{\mathrm{r}}+2 b \cdot \cos x_{\mathrm{r}} \cdot \sin x_{\mathrm{r}}+c \cdot \sin ^{2} x_{\mathrm{r}}
$$

hat die Koeffizienten

$$
\begin{aligned}
& a=\operatorname{SDj}^{2} x_{\mathrm{i}}\left(n_{\mathrm{r}}{ }^{2}+n_{\mathrm{i}}^{2}\right) \cdot \alpha+\operatorname{Sin}^{2} x_{\mathrm{i}} \cdot \beta-2 \operatorname{Evj} x_{\mathrm{i}} \cdot \operatorname{Sin} x_{\mathrm{i}}\left(n_{\mathrm{r}} W+n_{\mathrm{i}} V\right), \\
& c=\operatorname{Sofj}^{2} x_{\mathrm{i}} \cdot \beta+\operatorname{Sin}^{2} x_{\mathrm{i}} \cdot\left(n_{\mathrm{r}}^{2}+n_{\mathrm{i}}^{2}\right) \cdot \alpha-2 \operatorname{Evj} x_{\mathrm{i}} \cdot \operatorname{Sin} x_{\mathrm{i}}\left(n_{\mathrm{r}} W+n_{\mathrm{i}} V\right), \\
& b=-n_{\mathrm{r}} V+n_{\mathrm{i}} W
\end{aligned}
$$

und ist wegen $a c-b^{2}>0$ und $a>0$ positiv definit.

Die Größen $W$ und $V$ sind bereits in (17) und (18) erklärt. Spaltet man auch die Energieausdrücke (16) und (22) in Real- und Imaginärteil auf, so zeigt ein Vergleich mit (44), daß jeweils der erste Term von Real- und Imaginärteil des Spulenwiderstands der Strahlung ins Vakuum entspricht und der zweite Term der Einwirkung auf das Plasma.

Eine hinreichend genaue Übersicht über den Verlauf von (44) kann leider nur auf numerischem Wege gewonnen werden. Die Auswertung erfolgte im Rahmen einer Proberechnung auf der Göttinger Rechenmaschine G 1 a, welche Rechnungen mit gleitendem Komma ermöglicht.

Falls $\left|n_{\mathrm{i}}\right| \ll n_{\mathrm{r}}$ ist, kann man bei Änderung der Frequenz $\omega$ die Variation $\Delta\left(n_{\mathrm{i}} k R\right)$ gegen $\Delta\left(n_{\mathrm{r}} k R\right)$ vernachlässigen und innerhalb gewisser Bereiche den Widerstand $\Re$ als nur von $x_{\mathrm{r}}$ abhängig betrachten, während $x_{\mathrm{i}}$ konstant bleibt. Auf diese Weise wurde eine Übersicht über die Abhängigkeit von der Teilchendichte, dem Magnetfeld, der Temperatur und der Frequenz gewonnen. Abb. 5 zeigt den Realteil des Eingangswiderstandes einer mit DeuteriumPlasma gefüllten HF-Spule als Funktion der Phase $x_{\mathrm{r}}$ am Plasmarand. Das statische Magnetfeld beträgt $10^{2}$ Gauss, die Teilchendichte $10^{14} \mathrm{~cm}^{-3}$ und die Temperatur $10^{7} \mathrm{~K} \triangleq 1 \mathrm{keV}$. Scharparameter ist das relative Frequenzquadrat $x=\left(\omega / \omega_{0}\right)^{2}$. Jedes Maximum entspricht der Frequenz, die einer komplexen Eigenfrequenz des Systems am nächsten liegt. Man erkennt ein Abflachen der Welligkeit mit zunehmender Frequenz. Eine genaue Analyse von (44) zeigt, daß die Welligkeit $\eta$ im wesentlichen abfällt wie $n_{\mathrm{i}}{ }^{-2}$.

$$
\begin{gathered}
\eta=\frac{\operatorname{Max} \cdot \mathfrak{R e}\{\mathfrak{R}\}}{\operatorname{Min} \cdot \mathfrak{R e}\{\mathfrak{R}\}} \approx{n_{\mathrm{i}}}^{-2}, \\
n_{\mathrm{i}}=\text { const } \cdot \omega\left(\frac{N}{{H_{0}{ }^{2} T(1-x)}^{2}}\right)^{3 / 2} .
\end{gathered}
$$

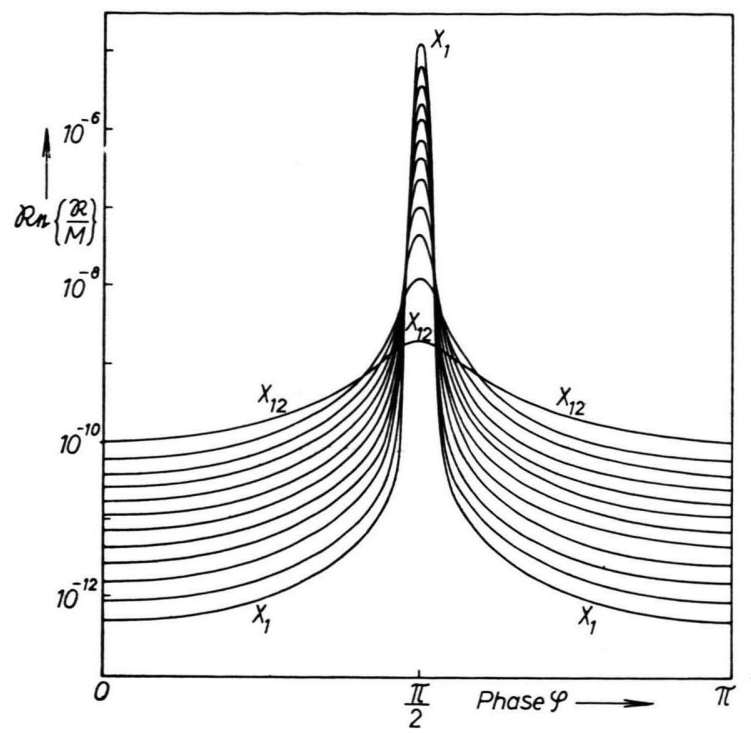

Abb. 5.

Frequenzganz von $\mathfrak{R e}\{\mathfrak{R} / M\}$; Scharparameter $x=\left(\omega / \omega_{0}\right)^{2}$. $H_{0}=10^{2} \mathrm{Gau} \beta ; \quad N=10^{14} \mathrm{~cm}^{-3} ; \quad T=10^{7}{ }^{\circ} \mathrm{K} ; \quad R=3 \mathrm{~cm}$.

$$
\begin{array}{ll}
x_{1}=0,010 . & x_{2}=0,018, x_{3}=0,031, \\
x_{4}=0,052, & x_{5}=0,083, x_{6}=0,129, \\
x_{7}=0,193, & x_{8}=0,279, x_{9}=0,387, \\
x_{10}=0,518, & x_{11}=0,669, x_{12}=0,833,
\end{array}
$$

Es nimmt also die Welligkeit unterhalb der Ionenresonanz $(x<1)$ mit steigender Frequenz und steigender Dichte $a b$, und mit steigender Temperatur und steigendem Magnetfeld zu. Für die Dichte der Extrema gelten die Bemerkungen über die Eigenwertdichte in II.

Abb. 6 zeigt in der komplexen Widerstandsebene die Ortskurven des Scheinwiderstandes der Plasmaspule. Um beide Vorzeichen von $\mathfrak{J} \mathfrak{m}\{\Re\}$ darstellen zu können, wurde in der logarithmischen Darstellung der Nullpunkt unterdrückt. Die Plasmadaten sind dieselben wie in Abb. 5. Längs jeder Kurve läuft als 
Kurvenparameter die Phase $\varphi=x_{\mathrm{r}}+\frac{\pi}{4}=n_{\mathrm{r}} k R$ der stehenden Welle am Plasmarand von 0 bis $\pi$. Die Änderung von $\varphi$ kann verursacht werden durch kleine Änderung der Plasmadaten oder der Frequenz. Die Kurven werden im Uhrzeigersinne durch-

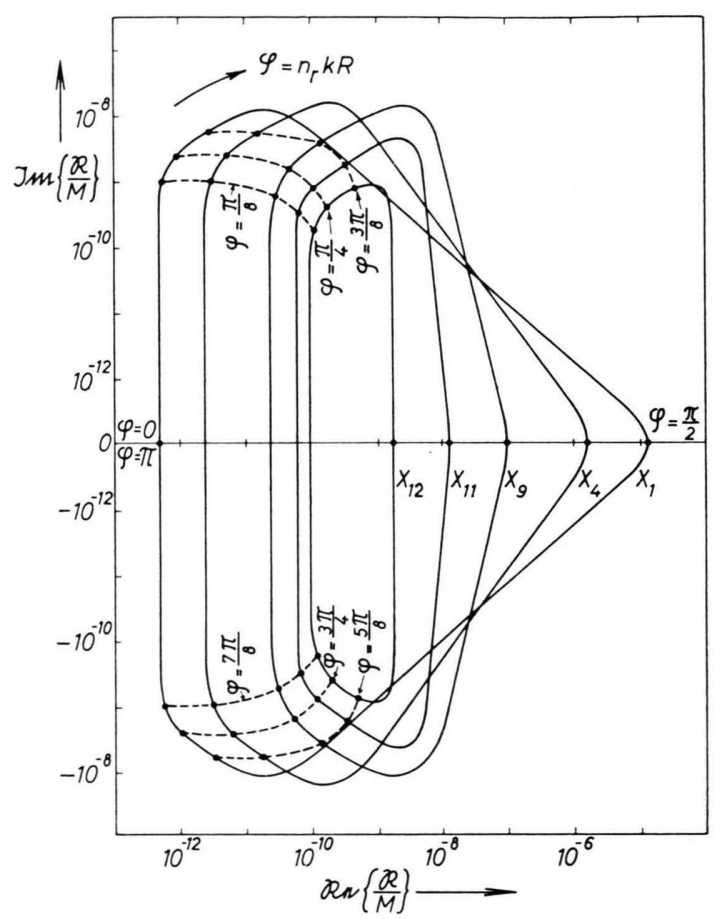

Abb. 6. Ortskurven der Eingangsimpedanz einer Plasmaspule. $H_{0}=10^{2} \mathrm{Gau} \beta ; \quad N=10^{14} \mathrm{~cm}^{-3} ; \quad T=10^{7}{ }^{\circ} \mathrm{K} ; \quad R=3 \mathrm{~cm}$.

laufen. Die fetten Punkte $\bullet$ markieren die Phasenlagen $\varphi=v \frac{\pi}{8},(v=0,1,2, \ldots)$. Die scharfe Spitze in $\Re e\{\Re\}$ (Abb. 5) äußert sich hier in dem großen Abstand der Marken in der Umgebung der Stelle $\varphi=\frac{\pi}{2}$. Außerdem springt hier der Imaginärteil von $\Re$ von positiven zu negativen Werten. Der Widerstand geht in der Nähe der Eigenresonanz des Plasmazylinders sehr schnell von induktiven zu kapazitiven Werten über. Mit steigender Frequenz wird die Schwankung des Realteils kleiner, während die des Imaginärteiles fast konstant bleibt. Bei anderen Plasmadaten dreht häufig in unmittelbarer Nähe der Ionenresonanz die Schwankung von $\Re \in\{\Re\}$ ihr Vorzeichen um. Die senkrechten Äste der Ortskurve durchdringen sich, so daß die Ortskurve von der Phase im Gegenuhrzeigersinn durchlaufen wird. Wegen der Dichte der Eigenwerte genügen schon Schwankungen der Plasmaparameter um wenige Prozent, um die ganze Kurve zu durchlaufen.

Genau dieselben Verhältnisse wie beim Widerstand treten auch bei den Leistungen $P_{1}$ (16) und $P_{2}$ (22) auf, da auch die Amplituden $a$ und $\alpha_{2}$ (21) und (15) den Resonanznenner (41) enthalten. Die Leistungsaufnahme des Plasmas hängt also entscheidend von der Anpassung des Wellenwiderstandes des Plasmas an den des Vakuums, d. h. von der Durchlässigkeit der Grenzfläche zwischen diesen beiden Medien ab. Dieses Ergebnis ist auch physikalisch plausibel, da sich die Verhältnisse qualitativ nicht ändern, wenn man die Stromschicht in einigen $\mathrm{Ab}$ stand vom Plasma ins Vakuum verlegt.

Praktisch ist aber eine solche Anpassung in den meisten Fällen durch Einstellen des günstigsten Zylinderradius, der Frequenz etc. wegen der großen Schärfe und Dichte der Resonanzen nicht erreichbar. Da technisch auch keine automatische Einregulierung optimaler Bedingungen möglich ist, und auch kein Mechanismus des schwingenden Systems bekannt ist, der evtl. gewisse Phasenlagen der stehenden Welle im Plasma bevorzugen würde, hat man sich auf eine pauschale Aussage zu beschränken, die man durch geeignete Mittelwertbildung über alle Phasenlagen gewinnt. Praktisch wird eine solche Mittelwertbildung während des Aufheizvorganges durch langsame Veränderung einiger Parameter des Plasmas von selbst durchgeführt.

Die betreffenden fastperiodischen Funktionen durchlaufen ihren Wertevorrat genau einmal, wenn sich bei konstanter Frequenz und Konstanz der Plasmadaten der Radius des Zylinders um einen Wert $\Delta R$ ändert, so daß $n \cdot \frac{\omega}{c} \cdot \Delta R$ ist. Nimmt man nun an, daß die Randverschiebung höchstens mit der mittleren thermischen Geschwindigkeit der Deuteronen erfolgen kann, dann ist die Mittelung sicher sinnvoll, solange die Schwingungszeit der Welle und damit des oszillierenden Zylinders klein ist gegen die für eine Veränderung von $R$ um $\Delta R$ durch thermische Bewegung erforderliche Zeit. Die Bedingung

$$
1 / \omega \ll \Delta R / v_{\mathrm{i}}
$$

ist erfüllt, falls $v_{\text {i }} n \ll \pi c$, oder falls $10^{1 / 2 \mu-\nu+1 / 2 r}$ $\ll 10^{15 / 2}$ ist mit

$$
\begin{gathered}
\mu=\log \left(N \mathrm{~cm}^{3}\right), \quad v=\log \left(H_{0} \mathrm{GAUSS}^{-1}\right), \\
\tau=\log \left(T^{\circ} \mathrm{K}^{-1}\right) .
\end{gathered}
$$

Um den Mittelwert zu bilden, bringt man den 
Ausdruck (31) für $\Re$ auf die Form

$$
\Re=(-i) M \frac{a \cdot \sin x_{\mathrm{r}}+\beta \cdot \cos x_{\mathrm{r}}}{a \cdot \sin x_{\mathrm{r}}+b \cdot \cos x_{\mathrm{r}}}
$$

Die Koeffizienten $\alpha, \beta, a, b$ sind komplex und hängen von $x_{\mathrm{i}}=n_{\mathrm{i}} k R$ und $k R$ ab. Falls $\left|n_{\mathrm{i}}\right| \ll n_{\mathrm{r}}$ ist, kann man diese Größen innerhalb eines gewissen Bereiches von $x_{\mathrm{r}}$ als konstant ansehen. Integration der dann streng periodischen Funktion $\Re\left(x_{\mathrm{r}}\right)$ liefert dann nach bekannten Methoden der Funktionentheorie den Ausdruck

$$
\Re^{\varphi}=\frac{1}{2 \pi} \int_{0}^{2 \pi} \Re\left(x_{\mathrm{r}}\right) \mathrm{d}\left(x_{\mathrm{r}}\right)=(-i) M \cdot \frac{\alpha+i \beta}{a+i b},
$$

da $a / b$ nicht reell ist und deshalb das trigonometrische Nennerpolynom in (55) in der komplexen Ebene eine Ellipse um die Singularität beschreibt. Da auch $\alpha / \beta$ nicht reell sein kann, wenn $n_{\mathrm{i}} \neq 0$ ist, gilt nach derselben Formel für den Leitwert (5) der Plasmaspule

$$
\overline{\mathcal{S}}^{\varphi}=\Re^{-1^{\varphi}}=\frac{1}{-i M} \cdot \frac{a+i b}{\alpha+i \beta}=\left(\bar{\Re}^{\varphi}\right)^{-1} .
$$

Auf Grund der mathematischen Struktur des Ausdruckes (31) ist also der reziproke Wert des mittleren Widerstandes gleich dem Mittel des Reziprokwertes ${ }^{7}$. Einsetzen in (56) ergibt

$$
\bar{\Re}^{\varphi}=(-i) M \frac{H_{1}^{(2)}(k R)}{H_{0}(2)(k R)-i n \cdot H_{1}^{(2)}(k R)},
$$

also formal denselben Ausdruck (40) wie im Fall $\left|x_{\mathrm{i}}\right| \gg 1$ (s. ebenfalls Anm. ${ }^{7}$ ).

Mit den Abkürzungen $\alpha, \beta$ (45), (46) und $W, V$ läßt sich (58) aufspalten in Real- und Imaginärteil:

$\frac{\Re^{\varphi}}{M}=\frac{\left(W+\alpha \cdot n_{\mathrm{r}}\right)-i\left(V+\alpha \cdot n_{\mathrm{i}}\right)}{\alpha\left(n_{\mathrm{r}}^{2}+n_{\mathrm{i}}^{2}\right)+\beta+2\left(n_{\mathrm{r}} W+n_{\mathrm{i}} V\right)} \approx \frac{1}{n_{\mathrm{r}}}-i \frac{V+\alpha \cdot n_{\mathrm{i}}}{\alpha \cdot n_{\mathrm{r}}{ }^{2}}$.

Weiter unterhalb der Ionenresonanz ist der Blindwiderstand also im Mittel kapazitiv; bei größeren $n_{\mathrm{i}}$ wird er induktiv.

\section{b) Ebenes Analogon}

Die Rückwirkung eines stehenden Wellenfeldes auf einen vorgegebenen Strom und damit auf die Leistungsabgabe des Stromes läßt sich am leichtesten am ebenen Modell studieren (Abb. 7).

In der Oberfläche $x=x_{0}$ eines dielektrischen Mediums mit dem Brechungsindex $n$ möge ein in $y$-Richtung fließender Wechselstrom

$$
j(x, y, z, t)=\left(0, \hat{I}_{y}, 0\right) \delta\left(x-x_{0}\right) e^{i \omega t}
$$

ebene elektromagnetische Wellen anregen, die in den Bereich $x>x_{0}$ hineinlaufen. An der Oberfläche $x=0$ möge eine Metallplatte die Wellen reflektieren, so daß sich in dem Dielektrikum stehende Wellen ausbilden. Das resultierende Wellenfeld ist dann mit dem identisch, welches ein bei $x=-x_{0}$ im Gegentakt

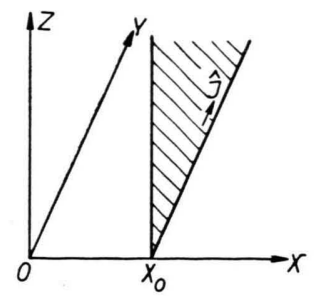

Abb. 7. Zum Strahlungswiderstand einer ebenen Stromschicht.

noch zusätzlich fließender Strom erzeugen würde. Die Phasenlage, die sich auf Grund der Plattendicke $x_{0}$ und der Wellenlänge am Rande $x=x_{0}$ einstellt, ist maßgebend für den in der Strombahn meßbaren Widerstand.

Die Felder lauten im Bereich $0 \leqq x \leqq x_{0}$ :

$$
\begin{gathered}
E_{y}=F \cdot \sin z \cdot e^{i \omega t}, \\
H_{z}=i n F \cdot \cos z \cdot e^{i \omega t}
\end{gathered}
$$

mit der Amplitude

$$
F=-\frac{4 \pi}{c} \cdot \hat{I}_{y} \cdot \frac{\sin z_{0}+i n \cos z_{0}}{\sin ^{2} z_{0}+n^{2} \cdot \cos ^{2} z_{0}}
$$

und der Phase $z=n k x, \quad z_{0}=n k x_{0}$.

Der Widerstand an der Stelle $x=x_{0}$ ergibt sich wieder aus energetischen Betrachtungen:

$$
\Re=\frac{4 \pi}{c} \cdot \frac{\sin z_{0}\left(\sin z_{0}+i n \cos z_{0}\right)}{1+\left(n^{2}-1\right) \cdot \cos ^{2} z_{0}} .
$$

Wenn $n$ reell ist, findet keine Absorption statt. Dann ist $\Re e\{\Re\}$ der Realteil des Strahlungswiderstandes und gibt ein $\mathrm{Ma}$ für die ins Vakuum abgestrahlte Leistung. Maximale Abstrahlung erfolgt, wenn das elektrische Feld am Rand einen Schwingungsbauch hat. Die Blindleistung verschwindet dann.

Wegen der Symmetrie von $\Im \mathfrak{m}\{\Re\}$ zur Stelle $z_{0}=\pi / 2$ wird der Blindwiderstand im Phasenmittel Null, da durch Krümmung der Stromfläche bedingte Unsymmetrien im $E$ - und $H$-Feld nicht auftreten (vgl. Abschn. 1). Der Blindwiderstand ist induktiv an Stellen, wo $\sin 2 z_{0}>0$. Wegen (61) und (62) läuft dort das Magnetfeld dem elektrischen Feld eine•

\footnotetext{
7 Dieses physikalisch sinnvolle Ergebnis kann auch als Rechtfertigung der Mittelung betrachtet werden.
} 
Viertelperiode voraus. [ Vgl. auch die Vakuumspule im quasistationären Grenzfall (34).] An Stellen kapazitiven Widerstandes ist es umgekehrt. Wenn $n$ komplex ist, hängt die zeitliche Phase zwischen $E$ und $H$ vom Ort ab. Falls $\left|n_{\mathrm{i}}\right| \ll n_{\mathrm{r}}$ ist, stellt der obige idealisierte Fall jedoch eine gute Näherung dar.

\section{c) Luftspalt zwischen Plasma und Strombahn}

Bislang wurde der Spulenstrom formal durch einen azimutalen Oberflächenstrom auf dem Mantel des Plasmazylinders ersetzt. Den Einfluß des Luftspaltes zwischen der Spule und der Plasmaoberfläche kann man untersuchen, indem man auch die in diesem Bereich sich ausbildenden stehenden transversalen Vakuumwellen

$$
\begin{aligned}
& E_{q}(\mathrm{r}, t)=\left[\alpha_{1} J_{1}(k r)+\beta_{1} N_{1}(k r)\right] e^{i \omega t}, \\
& H z(\mathrm{r}, t)=i\left[\alpha_{1} J_{0}(k r)+\beta_{1} N_{0}(k r)\right] e^{i \omega t}
\end{aligned}
$$

berücksichtigt und über die Grenzbedingungen an das Feld im Plasma (19), (20) und über die Sprungbedingungen am Ort des Stromes an das äußere Vakuumfeld (13), (14) anschließt.

Bedeutet $R$ den Radius des Plasmazylinders und $R_{1} \geqq R$ den Radius der zylindermantelförmigen Stromschicht, dann hat man für die Amplitude $a, \alpha_{1}$, $\beta_{1}, \alpha_{2}$ das Gleichungssystem:

$$
\begin{aligned}
a J_{1}(n k R)-\alpha_{1} J_{1}\left(k^{\prime} R\right)-\beta_{1} N_{1}(k R) & =0, \\
a n J_{0}(n k R)-\alpha_{1} J_{0}(k R)-\beta_{1} N_{0}(k R) & =0, \quad(69) \\
\alpha_{1} J_{1}\left(k R_{1}\right)+\beta_{1} N_{1}\left(k R_{1}\right)-\alpha_{2} H_{1}^{(2)}\left(k R_{1}\right) & =0, \quad(70) \\
\alpha_{1} J_{0}\left(k R_{1}\right)+\beta_{1} N_{0}\left(k R_{1}\right)-\alpha_{2} H_{0}^{(2)}\left(k R_{1}\right) & =-\frac{4 \pi}{c} \hat{I}_{\varphi} .
\end{aligned}
$$

Für die Berechnung des Widerstandes genügt die Kenntnis des Koeffizienten $\alpha_{2}$. Man erhält nach bekannten Methoden ("Cramersche Regel“" und LAPLACE-Entwicklung der entsprechenden Determinanten) für den Widerstand der Plasmaspule mit Luftspalt

$$
\begin{aligned}
\Re=(-i) 2 \pi R_{1} & \frac{4 \pi}{c} l \nu^{2} H_{1}{ }^{(2)}\left(k R_{1}\right) \\
& \times \frac{n J_{0}(n k R) D+J_{1}(n k R) B}{N},
\end{aligned}
$$

wobei

$$
\begin{aligned}
N=J_{1}(n k R)\left[H_{1}{ }^{(2)}\left(k R_{1}\right) A+H_{0}{ }^{(2)}\left(k R_{1}\right) B\right] & \\
& +n J_{0}(n k R)\left[H_{1}{ }^{(2)}\left(k R_{1}\right) C+H_{0}{ }^{(2)}\left(k R_{1}\right) D\right]
\end{aligned}
$$

und

$$
\begin{aligned}
& A=J_{0}\left(k R_{1}\right) N_{0}(k R)-J_{0}(k R) N_{0}\left(k R_{1}\right), \\
& B=J_{0}(k R) N_{1}\left(k R_{1}\right)-J_{1}\left(k R_{1}\right) N_{0}(k R), \\
& C=J_{1}(k R) N_{0}\left(k R_{1}\right)-J_{0}\left(k R_{1}\right) N_{1}(k R), \\
& \left.\left.D=J_{1}\left(k R_{1}\right) N_{1}\right) k R\right)-J_{1}(k R) N_{1}\left(k R_{1}\right)
\end{aligned}
$$

bedeuten. In der Grenze $R_{1} \rightarrow R$ folgt natürlich hieraus wieder den Ausdruck (31). Da in allen aus (72) entwickelten Näherungen das Ergebnis in bekannter Weise entscheidend von der Phasenlage der Welle am Plasmarand abhängt, wird der weiteren Untersuchung der Mittelwert $\bar{\Re}^{\varphi}$ zugrunde gelegt. Wenn man die Bessel-Funktionen entwickelt und im Falle $\left|x_{\mathrm{i}}\right| \ll 1$ die schnell oszillierenden Terme von den langsam variablen trennt, erhält der Widerstand die Form

$$
\begin{aligned}
\Re=(-i) 2 \pi R_{1} \frac{4 \pi}{c} l & v^{2} H_{1}{ }^{(2)}\left(k R_{1}\right) \\
& \cdot \frac{K_{1} \cdot \cos x_{\mathrm{r}}+K_{2} \cdot \sin x_{\mathrm{r}}}{L_{1} \cdot \cos x_{\mathrm{r}}+L_{2} \cdot \sin x_{\mathrm{r}}},
\end{aligned}
$$

wobei $K_{1}, K_{2}, L_{1}, L_{2}$ und $K_{1} / K_{2}, L_{1} / L_{2}$ komplex sind.

Innerhalb der Periode $\pi$ der Variablen $x_{\mathrm{r}}$ können die Koeffizienten als konstant angesehen werden. Wendet man wieder das Verfahren der komplexen Integration an, so gelten dieselben Überlegungen wie bei (56) und man erhält wegen

$$
K_{2}+i K_{1}=\left(\operatorname{Eoj} x_{\mathrm{i}}-\operatorname{Sin}_{\mathrm{i}}\right)(B+i n D)
$$

und

$$
\begin{array}{r}
L_{2}+i L_{1}=\left(\operatorname{Coj} x_{\mathrm{i}}-\operatorname{Sin} x_{\mathrm{i}}\right)\left[H_{1}{ }^{(2)}\left(k R_{1}\right)(A+i n C)\right. \\
+H_{0}{ }^{(2)}\left(k R_{1}\right)(B+i n D)
\end{array}
$$

für den Eingangswiderstand im Phasenmittel

$$
\begin{aligned}
& \Re^{\varphi}=(-i) 2 \pi R_{1} \frac{4 \pi}{c} l \nu^{2} H_{1}{ }^{(2)}\left(k R_{1}\right) \\
& \frac{B+i n D}{H_{1}(2)\left(k R_{1}\right) \cdot(A+i n C)+H_{0}{ }^{(2)}\left(k R_{1}\right) \cdot(B+i n D)} .
\end{aligned}
$$

Mit dem Ansatz $\quad R_{1}=R(1+\varepsilon), \quad \varepsilon \ll 1$

ergibt eine Störungsrechnung 1. Ordnung in $\varepsilon$ :

$$
\begin{aligned}
A \approx-\frac{2}{\pi} \cdot \varepsilon, \quad B & \approx \frac{2}{\pi} \cdot \frac{1}{k R} \cdot(1-\varepsilon), \\
C & \approx \frac{2}{\pi} \cdot \frac{1}{k R} ; \quad D \approx \frac{2}{\pi} \cdot \varepsilon, \\
H_{1}{ }^{(2)}\left(k R_{1}\right) & \approx H_{1}{ }^{(2)}(k R)+\varepsilon \cdot\left(\frac{k R}{2}-i \frac{2}{\pi} \cdot \frac{1}{k R}\right), \\
H_{0}{ }^{(2)}\left(k R_{1}\right) & \approx H_{0}{ }^{(2)}(k R)-\varepsilon i \frac{2}{\pi} .
\end{aligned}
$$


Daraus folgt dann für die relative Änderung von $\bar{\Re}^{\varphi}$

$$
\frac{\Delta \overline{\mathfrak{R}}^{\varphi}}{\overline{\mathfrak{R}}^{\varphi}} \approx \varepsilon(1-i n k R)
$$

und für die absolute Änderung

$$
\Delta \bar{\Re}^{\varphi} \approx-i \varepsilon M k R \text {. }
$$

Radiusänderungen der Stromwindung wirken sich also wegen $n k R \gg 1$ relativ stark aus. Bei einer Vergrößerung des Radius wird der mittlere Widerstand kapazitiver; der Realteil bleibt unverändert. Die Leistungsaufnahme der Spule und damit die Summe der abgestrahlten und absorbierten Wirkleistung bleibt also konstant.

\title{
Hodhfrequenzheizung eines Plasmazylinders in einem axialen Magnetfeld*
}

\author{
Von KLaus Körper \\ Aus dem Max-Planck-Institut für Physik, München \\ (Z. Naturforschg. 15 a, 235-243 [1960]; eingegangen am 17. Januar 1960)
}

\begin{abstract}
The conditions under which the effect of ion-electron-scattering on radial oscillations near the ion-resonance of an infinite plasma cylinder in an axial magnetic field may lead to a heating of the plasma, possibly sufficient to induce thermonuclear reaction, are examined.

The magnetic field strengths needed to stabilize the plasma, and the density of the plasma necessary to guarantee a sufficient rate of reaction require that we consider the optical behaviour in the decimeter-wavelength-range.

In an equivalent model for the supplying circuit the reaction of the plasma is taken into account in a quantitative manner.

Because of the finite extent of the plasma a dense spectrum of eigenresonances of the plasma system exists. Therefore the matching of the external circuit to the plasma is possible only in the mean.

Numerical results are given for the energy absorbed, the radiative energy penetrating the plasma cylinder, and the corresponding reactive output, as functions of the plasma density, the temperature and the frequency (near the "ion-resonance" = geometric mean value between the gyrofrequencies of the ions and electrons), when the oscillator and the plasma are optimally matched (Fig. 4, 5, 6).
\end{abstract}

In früheren Arbeiten ${ }^{1,2}$ sind die radialen Schwingungen eines unendlich langen homogenen Plasmazylinders in einem axialen homogenen Magnetfeld untersucht worden, welche durch azimutale, eine HFSpule repräsentierende Oberflächenströme angeregt werden. Aus energetischen Betrachtungen des Strahlungsfeldes des schwingenden Plasmazylinders ließ sich die Eingangsimpedanz der Spule herleiten ${ }^{3}$. Dabei wurde der Oberflächenstrom in ein System von linearen Strömen aufgelöst und die Spulen- und Zylinderlänge auf einen endlichen Wert begrenzt, aber die Zylindersymmetrie beibehalten.

Ziel dieser Untersuchung ist die Entscheidung, bis zu einem welchen Grade Energie aus der elektromagnetischen Welle vom Plasma absorbiert wird. Dabei wird sich eine Energiebildung im wesentlichen auf einen Vergleich der in einem Plasma endlicher

\footnotetext{
* Auszugsweise vorgelegt auf der Intern. Konferenz über die friedliche Anw. der Atomenergie, Genf, Sept. 1958. Vorgetragen auf der Tagung der Physikalischen Gesellschaft in Bayern, in München, am 29. 4. 1959, und auf der Plasmatagung, T.H. Aachen, am 26. 10. 1959.
}

Lineardimension absorbierten Leistung zu der von der das Plasma durchdringenden Welle mitgeführten Energie stützen, denn andere, evtl. auftretende Energieverluste sind mehr durch die spezielle technische Anordnung als durch die Eigenschaften des Plasmas bedingt, und lassen sich im Prinzip zu Null machen. Sie werden daher nur sehr pauschal abgeschätzt werden können.

Auf diese Weise werden einige wesentliche Terme der allgemeinen Differentialgleichung bestimmt, welche während der Aufheizphase den zeitlichen Verlauf des Energieinhaltes und damit der Temperatur des Plasmas festlegt. Zur Integration wäre allerdings ein weiterer Einblick in die nicht berücksichtigten Verlustprozesse, z. B. mikroskopische Strahlung der Plasmamaterie, oder Korpuskularstrahlung aus dem Plasma heraus nötig.

1 K. KöRPER, Z. Naturforschg. 12 a, 815 [1957]. Im folgenden mit I zitiert.

2 K. KöRPER, Z. Naturforschg. 15 a, 220 [1960]. Im folgenden mit II zitiert.

3 K. KöRPER, Z. Naturforschg. 15 a, 226 [1960]. Im folgenden mit III zitiert. 